\title{
Nonsingular Models of Universes in Teleparallel Theories
}

\author{
Jaume de Haro* and Jaume Amoros ${ }^{\dagger}$ \\ Departament de Matemàtica Aplicada I, Universitat Politècnica de Catalunya, Diagonal 647, 08028 Barcelona, Spain
} (Received 27 November 2012; published 14 February 2013)

\begin{abstract}
Different models of universes are considered in the context of teleparallel theories. Assuming that the universe is filled by a fluid with an equation of state $P=-\rho-f(\rho)$, for different teleparallel theories and different equation of state we study its dynamics. Two particular cases are studied in detail: in the first one we consider a function $f$ with two zeros (two de Sitter solutions) that mimics a huge cosmological constant at early times and a pressureless fluid at late times; in the second one, in the context of loop quantum cosmology with a small cosmological constant, we consider a pressureless fluid $(P=0 \Leftrightarrow$ $f(\rho)=-\rho$ ) which means there are de Sitter and anti-de Sitter solutions. In both cases one obtains a nonsingular universe that at early times is in an inflationary phase; after leaving this phase, it passes trough a matter dominated phase and finally at late times it expands in an accelerated way.
\end{abstract}

DOI: 10.1103/PhysRevLett.110.071104

PACS numbers: $04.50 . \mathrm{Kd}, 98.80 . J \mathrm{~J}$

Introduction.-Teleparallel theories $(F(T)$ theories) [1-4] are built from the scalar torsion $T=-6 H^{2}$, where $H$ is the Hubble parameter. The field equations are secondorder, which is a great advantage to $F(R)$ theories, whose fourth-order equations lead to pathologies like instabilities or large corrections to Newton's law $[5,6]$. This entails that in teleparallel theories the modified Friedmann equation depicts a curve in the plane $(H, \rho)$, that is, the universe moves along this curve and its dynamics is given by the modified Raychaudhuri equation and the conservation equation.

This opens the possibility to build nonsingular models of universes filled by a fluid with equation of state (EOS) $P=$ $-\rho-f(\rho)$, with $P$ the pressure and $\rho$ the energy density. For this EOS, the zeros of the function $f$ give de Sitter and anti-de Sitter solutions. Then, choosing a function $f$ with two zeros, one obtains a nonsingular model of a universe.

As examples of teleparallel theories we study Einstein Cosmology (EC) with and without a small cosmological constant, loop quantum cosmology (LQC) with and without a small cosmological constant, and the teleparallel version of the model $F(R)=\frac{R}{2}-\frac{\mu^{4}}{2 R}, R$ being the scalar curvature. For these teleparallel examples we consider models of EOS, which mimic a huge cosmological constant at early times and a pressureless fluid at late times, like $P=-\frac{\rho^{2}}{\rho_{i}}, \rho_{i}$ being the initial energy density of the universe.

We will see how these simple models could solve the socalled coincidence problem due to periods of acceleration of our universe; that is, they could mimic the evolution of a universe that begins in an inflationary phase, and, after leaving, it passes through a matter dominated one, and at late times enters in an accelerated phase.

We also study the specific case of a universe filled by a pressureless fluid $(P=0)$ in the context of LQC with a small cosmological constant. In that case, at early times the universe is in an anti-de Sitter phase; after leaving this phase it starts to accelerate (inflationary phase) leaving the contracting phase to enter in the expanding one (it bounces), then it starts to decelerate and enters in a matter dominated phase. Finally, at late times it enters in a de Sitter phase (late time cosmic acceleration).

In some sense our work seems a teleparallel version of the early idea that $F(R)$ cosmology could unify inflation with late cosmic acceleration [7] if one uses a function $F$ of the form $F(R)=\frac{1}{2}\left(R-\frac{a}{\left(R-\Lambda_{1}\right)^{n}}+b\left(R-\Lambda_{2}\right)^{m}\right)$, with $n, m$, $a, b, \Lambda_{1}, \Lambda_{2} \geq 0$ parameters. However, our point of view is different from the one adopted in current dark energy cosmology (see Ref. [8] for a recent review). Here, in models of oscillating dark energy, one takes an EOS of the form $P=\omega(t) \rho$ (see for example Refs. $[9,10]$ ), or a scalar field under the action of an oscillating potential [11]. It is also different from the current $F(T)$ or $F(R)$ theories where, in order to reconstruct models realizing cosmologies, the authors have to use very complicated functions $F$ (see, for example, Refs. [12-15]).

The units used in the Letter are $c=\hbar=8 \pi G=1$.

Teleparallel cosmological theories.-Teleparallel theories are based in the Weitzenböck space-time. To build this space-time one chooses a global system of four orthonormal vector fields $\left\{\mathbf{e}_{i}\right\}$ which are related with the vectors $\left\{\partial_{\mu}\right\}$ via the relation $\mathbf{e}_{i}=e_{i}^{\mu} \partial_{\mu}$. Then one introduces a covariant derivative $\nabla$ that defines absolute parallelism with respect to the global basis $\left\{\mathbf{e}_{i}\right\}$, that is $\nabla_{\nu} e_{i}^{\mu}=0$. From this, one obtains the metric Weitzenböck connection $\Gamma_{\mu \nu}^{\gamma}=e_{i}^{\gamma} \partial_{\nu} e_{\mu}^{i}$. (Note that this connection is metric, it satisfies $\left.\nabla_{\gamma} g_{\mu \nu}=0\right)$.

The Weitzenböck space-time has an identically vanishing curvature (the Riemann tensor vanishes), but it is not torsion free. One effectively has $T_{\mu \nu}^{\gamma}=\Gamma_{\nu \mu}^{\gamma}-\Gamma_{\mu \nu}^{\gamma} \neq 0$. Then, in order to build the Lagrangian in the Weitzenböck space-time, one has to introduce the contorsion tensor 


$$
K_{\gamma}^{\mu \nu}=-\frac{1}{2}\left(T_{\gamma}^{\mu \nu}-T_{\gamma}^{\nu \mu}-T_{\gamma}^{\mu \nu}\right)
$$

and the tensor

$$
S_{\gamma}{ }^{\mu \nu}=\frac{1}{2}\left(K_{\gamma}^{\mu \nu}+\delta_{\gamma}^{\mu} T_{\theta}^{\theta \nu}-\delta_{\gamma}^{\nu} T_{\theta}^{\theta \mu}\right),
$$

to construct the so-called scalar torsion

$$
T=S_{\gamma}{ }^{\mu \nu} T^{\gamma}{ }_{\mu \nu},
$$

and one can define modified teleparallel theories via a Lagrangian of the form $\mathcal{L}_{T}=V F(T)-V \rho$, where $V$ is the volume of the spatial part.

What is interesting here is that if one chooses the basis $\left\{\mathbf{e}_{0}=\partial_{0}, \mathbf{e}_{1}=a \partial_{1}, \mathbf{e}_{2}=a \partial_{2}, \mathbf{e}_{3}=a \partial_{3}\right\}$, then for the Friedmann-Lemaître-Robertson-Walker (FLRW) metric one obtains [16] $T=-6 H^{2}$.

The problematic point in teleparallelism is that it depends on the choice of the global basis. In the sense that, if one uses a local Lorentz transformation to transform the original global basis in another one, in general, one obtains another Weitzenböck connection and thus $T$ could change $[17,18]$, which does not happen in modified gravity $[F(R)$ theories] where the invariants do not depend on a global basis. However, for a FLRW metric, if we use local Lorentz transformations that only depend on the time, that is, of the form $\overline{\mathbf{e}}_{i}=\Lambda_{i}^{k}(t) \mathbf{e}_{k}$, then, even though the torsion tensor changes, the torsion scalar $T$ remains constant with a value of $T=-6 H^{2}$. From our point of view, this gives a consistency with teleparallel theories in cosmology, because $T$ is invariant from "isotropic and homogeneous" local Lorentz transformations.

General features in teleparallel theories.-For the flat FLRW space-time filled by a perfect fluid with EOS $P(\rho)=-\rho-f(\rho)$, teleparallel theories, are obtained from the Lagrangian

$$
\mathcal{L}=V F(T)-V \rho(V),
$$

where $\rho(V)$ is obtained from the conservation equation (first principle of thermodynamics)

$$
d(\rho V)=-P d V \Longleftrightarrow \frac{d \rho}{d V}=\frac{f(\rho)}{V} \Longleftrightarrow \dot{\rho}=3 H f(\rho) .
$$

The conjugate momentum is then given by $p_{V}=\frac{\partial \mathcal{L}}{\partial \dot{V}}=$ $-4 H F^{\prime}(T)$, and thus the Hamiltonian is

$$
\mathcal{H}=\dot{V} p_{V}-\mathcal{L}=\left(2 T F^{\prime}(T)-F(T)+\rho\right) V .
$$

In general relativity the Hamiltonian is constrained to be zero. [In $f(T)$ gravity the Hamiltonian is also constrained to be zero, this comes from the equation given by the variation of the action with respect to the vierbien $e_{i}^{\mu}$. Actually, the constraint comes from the $(0,0)$ component of this equation (see Eq. (9) of Ref. [15])]. This constraint leads to the modified Friedmann equation

$$
\rho=-2 F^{\prime}(T) T+F(T) \equiv G(T),
$$

which is a curve in the plane $(H, \rho)$.

Conversely, given a curve of the form $\rho=G(T)$ for some function $G$, it could be obtained from the modified Friedmann equation by choosing

$$
F(T)=-\frac{\sqrt{-T}}{2} \int \frac{G(T)}{T \sqrt{-T}} d T .
$$

The modified Raychaudhuri equation is obtained from the modified Friedmann equation taking its derivative with respect to the time, giving rise to the equation $\dot{H}=$ $-\frac{f(\rho)}{4 G^{\prime}(T)}$. Then, the dynamics of the universe is given by the modified Raychaudhuri equation and the conservation equation, i.e., by the system

$$
\dot{H}=-\frac{f(\rho)}{4 G^{\prime}(T)} \quad \dot{\rho}=3 H f(\rho),
$$

provided the universe moves along the curve $\rho=G(T)$.

What is important to stress here is that the critical points of the system are given by the solutions of the equation $f(\rho)=0$, that is, the de Sitter and anti-de Sitter solutions are the solutions of the equation $f(\rho)=0$. This gives us a simple way to build nonsingular models of universes: One has to choose a function $f$ with two zeros (two de Sitter solutions, or a de Sitter and an anti-de Sitter solution), then the universe will move from one to the other. To finish this section, we will introduce three parameters which are important in cosmology: (1). $\omega \equiv \frac{P}{\rho}$ is useful to classify the fluid that fills the universe (when $\omega<-1$ the fluid is called phantom, when $\omega=0$ dust, when $\omega=1 / 3$ radiation, ...). (2). $\omega_{\text {eff }} \equiv \frac{1}{3}\left(1-\frac{R}{3 H^{2}}\right)=-1-\frac{2 \dot{H}}{3 H^{2}}$ is related to the expansion of the universe. Actually, when $\omega_{\text {eff }}<-1 / 3$ (respectively $\omega_{\text {eff }}>-1 / 3$ ) the universe accelerates (respectively decelerates). (3). $\Omega \equiv \frac{\rho}{3 H^{2}}$ gives the amount of matter in the universe. For a general teleparallel theory $\rho=G(T)$ one has $\omega=-1-\frac{f(\rho)}{\rho}$ and

$$
\omega_{\text {eff }}=-1-\frac{f(\rho)}{T G^{\prime}(T)}, \quad \Omega=-\frac{2 \rho}{T} .
$$

Examples of teleparallel theories.-In this section we will study three important examples of teleparallel theories: Einstein cosmology, loop quantum cosmology (LQC) [19], and the teleparallel version of the $F(R)=\frac{R}{2}-\frac{\mu^{4}}{2 R}$ model [20,21].

Einstein cosmology: The first example of teleparallel theory is Einstein cosmology (EC), which is obtained from the Lagrangian

$$
\mathcal{L}_{E}=\frac{1}{2} R V-\rho(V) V,
$$

where $R=6\left(\dot{H}+2 H^{2}\right)$ is the scalar curvature.

Note that, the Lagrangian can be written as follows: $\mathcal{L}_{E}=3 \frac{d \dot{V}}{d t}+\overline{\mathcal{L}}_{E}$, where 


$$
\overline{\mathcal{L}}_{E}=\frac{1}{2} T V-\rho V .
$$

Then, since $\mathcal{L}_{E}$ and $\overline{\mathcal{L}}_{E}$ differ by a total derivative, one can conclude that they are equivalent.

In the EC equation (7) becomes the Friedmann equation

$$
\frac{1}{2} T+\rho=0 \Longleftrightarrow H^{2}=\rho / 3,
$$

which depicts a parabola in the plane $(H, \rho)$, that is, the evolution of the universe follows this parabola, and its dynamics is given by the system

$$
\dot{H}=\frac{f(\rho)}{2} \quad \dot{\rho}=3 H f(\rho) .
$$

Note that in EC one always has $\omega=\omega_{\text {eff }}(\rho)=-1-\frac{f(\rho)}{\rho}$ and $\Omega \equiv 1$. Once we have obtained the dynamics in EC we can study some examples of EOS. The simplest one is given by a fluid with $\omega=\omega_{0}$ constant, which means that $f(\rho)=-\left(1+\omega_{0}\right) \rho$, and thus, the only critical point of the system is $(0,0)$. In that case, it is trivial to show that for $\omega_{0}>-1$ the universe moves from $\rho=\infty$ to $\rho=0$ (big bang singularity). And for a phantom field $\left(\omega_{0}<-1\right)$ the universe moves from $\rho=0$ to $\rho=\infty$ (big rip singularity).

To finish EC we will build a cosmological model without singularities. One could do this introducing a small cosmological constant, namely, $\mu^{4}$, which could be done changing, in the Lagrangian (12), $\rho$ by $\rho+\mu^{4}$. Once we have introduced this small cosmological constant, we will choose a fluid that for large values of $\rho$ mimics a huge cosmological constant $\left(\Lambda=\rho_{i}\right)$, and for small values of $\rho$ becomes pressureless. An example of this kind of fluids is given by an EOS

$$
P=-\frac{\rho^{2}}{\rho_{i}} \Leftrightarrow \omega=-\frac{\rho}{\rho_{i}} \Leftrightarrow f(\rho)=-\rho\left(1-\frac{\rho}{\rho_{i}}\right) .
$$

Then, the system has two critical points $a_{f}=\left(\mu^{2} / \sqrt{3}, 0\right)$ and $a_{i}=\left(\sqrt{\mu^{4}+\rho_{i}} / \sqrt{3}, \rho_{i}\right)$. At the point $a_{i}$ one has $\omega=\omega_{\text {eff }}=-1$ and $\Omega \cong 1$ (de Sitter phase), at $a_{f}$ one has $\omega=\omega_{\text {eff }}=-1$ and $\Omega=0$ (de Sitter phase), and when $\mu^{4} \ll \rho \ll \rho_{i}$ one has $\omega=\omega_{\text {eff }} \cong 0$ and $\Omega \cong 1$ (matter dominated phase).

This model shows a universe evolving from an early inflationary phase (point $a_{i}$ ) to a late time accelerated expansion (point $a_{f}$ ) passing through a matter dominated phase.

Note that asymptotically, this model converges to the LCDM model, because at late times $\rho \rightarrow 0$ and, consequently, the cosmological constant $\mu^{4}$ dominates. Then the deceleration, the jerk, and snark parameters (see Ref. [12] for the definition of these parameters) converge, respectively, to $-1,1$, and $s=0$. Note also that the model may be solved analytically and then one could evaluate these parameters in the different phases in which our universe evolves.
Loop quantum cosmology: The main idea of LQC is that it assumes a discrete nature of space which leads, at the quantum level, to consider a Hilbert space where quantum states are represented by almost periodic functions of the dynamical part of the connection [22-24]. Unfortunately, the connection variable doesn't correspond to a welldefined quantum operator in this Hilbert space, what leads to re-express the gravitational part of the Hamiltonian in terms of almost periodic functions, which could be done from a process of regularization. This new regularized (effective) Hamiltonian introduces a quadratic modification $\left(\rho^{2}\right)$ in the Friedmann equation at high energies $[25,26]$, which gives rise to a bounce when the energy density becomes equal to a critical value of the order of the Planck energy density. This modified Friedmann equation depicts the following ellipse in the plane $(H, \rho)$

$$
H^{2}=\frac{\rho}{3}\left(1-\frac{\rho}{\rho_{c}}\right) \Longleftrightarrow \frac{H^{2}}{\rho_{c} / 12}+\frac{\left(\rho-\frac{\rho_{c}}{2}\right)^{2}}{\rho_{c}^{2} / 4}=1,
$$

where $\rho_{c} \equiv \frac{3}{\gamma^{2} \lambda^{2}}$ is the so-called critical density, with $\gamma \cong 0.2375$ being the Barbero-Immirzi parameter and $\lambda$ a parameter with dimensions of length, which is determined invoking the quantum nature of the geometry, that is, identifying its square with the minimum eigenvalue of the area operator in LQG, which gives as a result $\lambda \equiv \sqrt{\frac{\sqrt{3}}{4} \gamma}$ (see Ref. [26]).

The dynamics is now given in LQC by the system

$$
\dot{H}=\frac{f(\rho)}{2}\left(1-\frac{2 \rho}{\rho_{c}}\right) \quad \dot{\rho}=3 H f(\rho),
$$

where the first equation is the modified Raychaudhuri equation in LQC. The parameters $\omega_{\text {eff }}$ and $\Omega$ become

$$
\omega_{\mathrm{eff}}=-1-\frac{f(\rho)}{\rho} \frac{\rho_{c}-2 \rho}{\rho_{c}-\rho}, \quad \Omega=\frac{\rho_{c}}{\rho_{c}-\rho} .
$$

As a first example, we can study an EOS with $f(\rho)<0$ (nonphantom fluid because $\omega>-1$ ). Then one obtains a cyclic universe moving in an clockwise sense along the ellipse. This universe bounces at points $a_{1}=(0,0)$ and $a_{2}=\left(0, \rho_{c}\right)$. In $a_{1}$ the universe enters in the contracting phase and in $a_{2}$ the universe enters in the expanding one. On the other hand, if one considers a phantom fluid $f(\rho)>0$, one obtains a cyclic universe moving in an anticlockwise sense along the ellipse.

As a second example, we consider a fluid with $\omega=\omega_{0}$ constant, which means that $f(\rho)=-\left(1+\omega_{0}\right) \rho$, and thus, the only critical point of the system is $(0,0)$. In this situation, it is easy to show that for $\omega_{0}>-1$ the universe moves from $(0,0)$ to itself in a clockwise sense, and for a phantom fluid $\left(\omega_{0}<-1\right)$ the universe moves from $(0,0)$ to itself in an anticlockwise sense.

The third example is more interesting because we will build a model without singularities. To do this we perform a small modification in the modified Friedmann equation, 
introducing a small cosmological constant $\mu^{4}$ [changing $\rho$ by $\rho+\mu^{4}$ in Eq. (16)] satisfying $\mu^{4} \ll \rho_{c}$. Then, Eq. (16) becomes the ellipse

$$
H^{2}=\frac{\rho+\mu^{4}}{3}\left(1-\frac{\rho+\mu^{4}}{\rho_{c}}\right) .
$$

For a pressureless fluid $[P=0 \Leftrightarrow f(\rho)=-\rho]$, the system has two critical points $a_{f} \equiv\left[\frac{\mu^{2}}{\sqrt{3}} \sqrt{\left(1-\frac{\mu^{4}}{\rho_{c}}\right)}, 0\right]$ and $a_{i} \equiv$ $\left[-\frac{\mu^{2}}{\sqrt{3}} \sqrt{\left(1-\frac{\mu^{4}}{\rho_{c}}\right)}, 0\right]$. The first one is a de Sitter solution and the second one is an anti-de Sitter solution. In that case the universe moves along the ellipse from $a_{i}$ to $a_{f}$ in a clockwise sense. When it arrives at the point $a_{1} \cong$ $\left(-\rho_{c} / 4, \rho_{c} / 4\right)$ the universe starts to accelerate (inflationary phase) because $\frac{\ddot{a}}{a}=\dot{H}+H^{2} \geq 0$ when $\rho \geq \rho_{c} / 4$; then it bounces at the top of the ellipse; i.e., at the point $a_{2}=$ $\left(\rho_{c}-\mu^{4}, 0\right)$ the universe leaves the contracting phase and enters the expanding one where the energy density starts to decrease. When the universe arrives at $a_{3} \cong\left(\rho_{c} / 4, \rho_{c} / 4\right)$ it starts to decelerate and when the density satisfies $\rho_{c} \ll$ $\rho \ll \mu^{4}$ the universe enters in a matter dominated phase $\left(\omega_{\text {eff }} \cong 0\right.$ and $\left.\Omega \cong 1\right)$. Finally, after leaving this phase it goes asymptotically, at late times, to the point $a_{f}$ (de Sitter phase that mimics the late time accelerated cosmic expansion).

Note that a model of a universe moving from an inflationary phase towards a late time accelerating phase passing through a matter dominated phase, could also be obtained in LQC with a small cosmological constant by choosing as EOS of the model given in (15), with $\rho \approx$ $\rho_{c} / 2$. For this EOS, the universe moves from the de Sitter solution $a_{i}=\left[\sqrt{\frac{\rho_{i}+\mu^{4}}{3}\left(1-\frac{\rho_{i}+\mu^{4}}{\rho_{c}}\right)}, \rho_{i}\right]$ satisfying $\omega=$ $\omega_{\text {eff }}=-1$ and $\Omega=2$, to the de Sitter one $a_{f} \cong\left(\frac{\mu^{2}}{\sqrt{3}}, 0\right)$ satisfying $\omega=\omega_{\text {eff }}=-1$ and $\Omega=0$.

A teleparallel version of the $F(R)=\frac{R}{2}-\frac{\mu^{4}}{2 R}$ model: As a last example of teleparallel theory, we consider the function $G(T)=-\frac{T}{2}+\frac{3 \mu^{4}}{2 T}$, which corresponds to the model $F(T)=\frac{T}{2}+\frac{\mu^{4}}{2 T}$ [the teleparallel version of $\left.F(R)=\frac{R}{2}-\frac{\mu^{4}}{2 R}\right]$. In this case one has

$$
\omega_{\text {eff }}=-1-\frac{f(\rho)}{\sqrt{\rho^{2}+3 \mu^{4}}}, \quad \Omega=\frac{2 \rho}{\rho+\sqrt{\rho^{2}+3 \mu^{4}}} .
$$

Then, for a universe filled by a pressureless fluid $P=0$ [i.e., $f(\rho)=-\rho$ ], when $\rho$ takes large values one has $\omega_{\text {eff }} \cong 0, \Omega \cong 1$, and for small values of $\rho$ one has $\omega_{\text {eff }} \cong$ $-1, \Omega \cong 0$. This means that, at early times $(\rho \rightarrow \infty)$ the universe is in the matter dominated phase, and at the late times $(\rho=0)$ it enters in the de Sitter phase. The universe accelerates and is not singular at late times, but at early times it is singular. This could be easily deduced because the universe is in the matter dominated phase at early times, or from the conservation equation which for large values of $\rho$ reads $\dot{\rho} \cong-\sqrt{3} \rho^{3 / 2}$. Then, at early times, one gets

$$
H(t) \cong \frac{2}{3\left(t-t_{s}\right)}, \quad \rho(t) \cong \frac{4}{3\left(t-t_{s}\right)^{2}} .
$$

Finally, we look for a universe without singularities. Once again we consider the EOS (15) with $\rho_{i} \gg \sqrt{3} \mu^{2}$ which leads to a dynamical system with two critical points (two de Sitter solutions) at $a_{f} \equiv\left(\frac{\sqrt{3} \mu^{2}}{6}, 0\right)$ and $a_{i} \equiv\left(\frac{\rho_{i}+\sqrt{\rho_{i}^{2}+3 \mu^{4}}}{6}, \rho_{i}\right)$. Note that, for this model, we do not need to introduce any small cosmological constant because it is implicitly contained in the model.

At the de Sitter points $\omega_{\text {eff }}$ equals to -1 and $\Omega$ take the values

$\Omega\left(a_{f}\right)=0 \quad$ and $\quad \Omega\left(a_{i}\right)=\frac{2 \rho_{i}}{\rho_{i}+\sqrt{\rho_{i}^{2}+3 \mu^{4}}} \cong 1$,

and when $\sqrt{3} \mu^{2} \ll \rho \ll \rho_{i}$, one has $\omega_{\text {eff }} \cong 0$ and $\Omega \cong 1$, that is, the universe is in the matter dominated phase, which means that the universe moves from the point $a_{i}$ to the point $a_{f}$ along the curve $\rho=-\frac{T}{2}+\frac{3 \mu^{4}}{2 T}$ passing through a matter dominated phase.

The authors would like to thank Professor Sergei D. Odintsov for his valuable and useful comments. This investigation has been supported in part by MICINN (Spain), projects MTM2008-06349-C03-01 and MTM2009-14163-C02-02, and by AGAUR (Generalitat de Catalunya), Contracts No. 2009SGR 345 and No. 994.

*jaime.haro@upc.edu †jaume.amoros@upc.edu

[1] F. W. Hehl, P. Von Der Heyde, G. D. Kerlick, and J. M. Nester, Rev. Mod. Phys. 48, 393 (1976).

[2] K. Hayashi and T. Shirafuji, Phys. Rev. D 19, 3524 (1979); 24, 3312 (1982).

[3] E. E. Flanagan and E. Rosenthal, Phys. Rev. D 75, 124016 (2007); 75, 124016 (2007).

[4] J. Garecki, arXiv:1010.2654.

[5] S. Nojiri and S.D. Odintsov, Phys. Lett. B 652, 343 (2007).

[6] E. V. Linder, Phys. Rev. D 81, 127301 (2010).

[7] S. Nojiri and S.D. Odintsov, Phys. Rev. D 68, 123512 (2003).

[8] K. Bamba, S. Capozziello, S. Nojiri, and S. D. Odintsov, Astrophys. Space Sci. 342, 155 (2012).

[9] S. Nojiri and S. D. Odintsov, Phys. Lett. B 637, 139 (2006).

[10] B. Feng, M. Li, Y. Piao, and X. Zang, Phys. Lett. B 634, 101 (2006).

[11] S. Dodelson, M. Kaplinghat, and E. Stewart, Phys. Rev. Lett. 85, 5276 (2000).

[12] K. Bamba, R. Myrzakulov, S. Nojiri, and S. D. Odintsov, Phys. Rev. D 85, 104036 (2012). 
[13] S. Nojiri and S. D. Odintsov, Phys. Lett. B 657, 238 (2007).

[14] G. Cognola, E. Elizalde, S. Nojiri, S. D. Odintsov, L. Sebastiani, and S. Zerbini, Phys. Rev. D 77, 046009 (2008).

[15] Y.-F. Cai, S.-H. Chen, J.B. Dent, S. Dutta, and E. N. Saridakis, Classical Quantum Gravity 28, 215011 (2011).

[16] G. R. Bengochea and R. Ferraro, Phys. Rev. D 79, 124019 (2009).

[17] B. Li, T. P. Sotiriou, and J. D. Barrow, Phys. Rev. D 83, 064035 (2011).

[18] M. Li, R. X. Miao, and Y. G. Miao, J. High Energy Phys. 07 (2011) 108.

[19] K. Bamba, J. de Haro, and S. D. Odintsov, arXiv:1211.2968.
[20] S. M. Carroll, V. Duvvuri, M. Trodden, and M. S. Turner, Phys. Rev. D 70, 043528 (2004).

[21] S. M. Carroll, A. De Felice, V. Duvvuri, D. A. Easson, M. Trodden, and M.S. Turner, Phys. Rev. D 71, 063513 (2005).

[22] A. Ashtekar, M. Bojowald, and J. Lewandowski, Adv. Theor. Math. Phys. 7, 233 (2003).

[23] A. Ashtekar and P. Singh, Classical Quantum Gravity 28, 213001 (2011).

[24] A. Ashtekar, Nuovo Cimento Soc. Ital. Fis. B 122, 135 (2007).

[25] P. Singh, K. Vandersloot, and G. V. Vereshchagin, Phys. Rev. D 74, 043510 (2006).

[26] P. Singh, J. Phys. Conf. Ser. 140, 012005 (2009). 